\title{
Exchange-correlation effects on quantum wires with spin-orbit interactions under the influence of in-plane magnetic fields
}

\author{
Francesc Malet, Martí Pi, and Manuel Barranco \\ Departament ECM, Facultat de Física, and $\mathrm{IN}^{2}$ UB, Universitat de Barcelona, Diagonal 647, 08028 Barcelona, Spain \\ Llorenç Serra \\ Departament de Física and Institut Mediterrani d'Estudis Avançats IMEDEA (CSIC-UIB), Universitat de les Illes Balears, \\ 07122 Palma de Mallorca, Spain \\ Enrico Lipparini \\ Dipartimento di Fisica, Università di Trento, 38050 Povo, Trento, Italy \\ and INFN, via Enrico Fermi, 40-00044 Frascati, Rome, Italy
}

(Received 20 April 2007; published 10 September 2007)

\begin{abstract}
Within the noncollinear local spin-density approximation, we have studied the ground state structure of a parabolically confined quantum wire submitted to an in-plane magnetic field, including both Rashba and Dresselhaus spin-orbit interactions. We have explored a wide range of linear electronic densities in the weak (strong) coupling regimes that appear when the ratio of spin-orbit to confining energy is small (large). These results are used to obtain the conductance of the wire. In the strong coupling limit, the interplay between the applied magnetic field-irrespective of the in-plane direction, the exchange-correlation energy, and the spinorbit energy - produces anomalous plateaus in the conductance vs linear density plots that are otherwise absent, or washes out plateaus that appear when the exchange-correlation energy is not taken into account.
\end{abstract}

DOI: 10.1103/PhysRevB.76.115306

\section{INTRODUCTION}

The possibility to carry out controllable manipulations of electron spins using electric fields is the main goal of spintronics. This emerging field, based on the spin-orbit (SO) interaction, exploits the spin rather than the electron charge for information processing and promises remarkable new devices, faster, smaller, and more powerful than those currently existing. This has prompted an intense activity in the study of semiconductor heterostructures, since they present intrinsic SO interactions due to the existence of macroscopic electric fields arising from inversion asymmetry properties characteristic of those systems, which give rise to the Rashba SO coupling (related to the inversion asymmetry of the confining potential in the growing direction) and Dresselhaus SO coupling (related to the bulk inversion asymmetry). Among these nanostructures, quantum wires (QWs) are especially well suited for the development of spintronic devices. On the one hand, their transverse length can be externally controlled, making the system more or less quasi-onedimensional and, hence, changing the ratio of the SO strength to the confinement. On the other hand, the electron motion can be rendered almost collisionless because of the high purity of the starting two-dimensional electron gas.

The energy subband structure and conductance $(G)$ of QWs including SO effects have been addressed by several authors. Most of them have only taken into account the Rashba coupling ${ }^{1-3}$ because it can be tuned using gate voltages. Contrarily, the Dresselhaus coupling is fixed since it arises from basic properties of the semiconductor crystal. Also, the effect of applied magnetic fields $(B)$, either in or perpendicular to the plane containing the QW, has been considered in combination with only the Rashba ${ }^{4-6}$ or both ${ }^{7} \mathrm{SO}$ interactions.
PACS number(s): 73.63.Nm, 71.70.Ej, 71.15.Mb, 71.70.Gm

Interesting features of the energy subbands and $G$ have been disclosed, especially for strong SO couplings and in combination with magnetic fields applied to the QW. Among them, the presence of anticrossings, $k$ asymmetries, local extrema, and energy gaps in the subband spectra, or the socalled anomalous plateaus in the conductance, are some of the most interesting. By anomalous plateaus (or steps) we mean those appearing in the conductance that do not follow the increasing step sequence in units of $G_{0}=2 e^{2} / \hbar$, which pertains to the Landauer formula. The importance of taking into account the Rashba intersubband coupling term, neglected in some works, has also been pointed out. ${ }^{4}$

In the above mentioned works, the electron-electron interaction has not been taken into account. It is, thus, worthy to elucidate to which extent these results change when this interaction is considered, at least in a workable and sound mean field approximation, and this is the purpose of this paper. We address here the effects of the exchangecorrelation interaction on the energy subband structure and conductance of a quantum wire within the noncollinear local-spin density approximation (LSDA). To this end, we implement a self-consistent solution of the Kohn-Sham equations for a $\mathrm{QW}$ of length $L$ submitted to a parabolic lateral confinement, including SO effects and an in-plane magnetic field applied either parallel or perpendicular to the longitudinal axis of the wire. This gives the wave functions and the energy spectra of the QW, which, in turn, are used to calculate the conductance and other properties of the system, such as the phase diagram-energy per electron vs linear density - and the spin textures that appear across the wire. This work extends our results for a wire in the LSDA (without SO) (Ref. 8) and for a noninteracting wire with Rashba SO interaction. ${ }^{4}$

To avoid the cumbersome evaluation of the band occupations at zero temperature $(T)$, we use a finite- $T$ formalism. 
More specifically, the calculation of the "turning points" where the electron chemical potential crossing the energy subbands becomes involved when the bands are not fully parabolic. This is the case when SO terms and magnetic fields are included. The finite- $T$ frame is used here as a numerical trick, since in practice we have chosen $T$ small enough so that our results are $T=0$ results. Thermal effects may be introduced, increasing the value of $T$ in the relevant expressions, but we have not considered this possibility here.

This work is organized as follows. In Sec. II, we introduce the system and its variables, the Hamiltonian and the noncollinear LSDA needed to describe it, as well as examples of spin textures and energy subband structures of the QW obtained for some selected values of the model parameters, i.e., intensity and direction of $B$, and strength of the SO interaction with respect to the value of the lateral confining energy. The conductance of the QW in the weak and strong SO coupling regimes is discussed in Sec. III, together with an alternative derivation, based on the application of linear response theory, of the conductance of a $\mathrm{QW}$, equivalent to the one introduced in Ref. 9. A summary is given in Sec. IV, and we discuss in an appendix the subband structure up to second order in perturbation theory when the exchange-correlation energy is neglected. The first-order expression is a useful guide to understand some aspects of the complete, numerical calculation.

\section{FORMALISM}

\section{A. System and its variables}

We consider a parabolic confinement in the $y$ direction and free motion along the $x$ direction. The electrons are treated within the effective-mass, dielectric constant model in two dimensions, with the motion restricted to the $x y$ plane. Although no structure is strictly two-dimensional, it is commonly accepted that the confinement in the perpendicular direction is so strong that two-dimensional models catch the basic physics of the processes under study while they render the numerical effort much more affordable. The vertical extension of the structure is phenomenologically taken into account in the effective values of the spin-orbit constants, but it is definitely missing in the electron exchange-correlation energy used to address the problem.

The SO terms prevent the formation of good spin states. Therefore, the Kohn-Sham (KS) orbitals are two-component spinors of the type

$$
\Psi_{n k}(\mathbf{r}) \equiv\left|\Psi_{n k}\right\rangle \equiv \frac{1}{\sqrt{L}}\left(\begin{array}{c}
\varphi_{n k}(y, \uparrow) \\
\varphi_{n k}(y, \downarrow)
\end{array}\right) e^{i k x} .
$$

Translational invariance along $x$ allows us to introduce a continuous wave number $k$, and the index $n=1,2,3, \ldots$, labels the different energy subbands. Therefore, the quantum labels are $(n, k)$. This implies that we have no spin label for the subbands, and each of them contains both spin components and satisfies a Kohn-Sham spinorial equation

$$
h_{K S}[\rho, \mathbf{m}]\left|\Psi_{n k}\right\rangle=\varepsilon_{n k}\left|\Psi_{n k}\right\rangle .
$$

In Eq. (2), we have a functional dependence on electron density $\rho$ and spin magnetization $\mathbf{m}$, the latter being a vector. In order to determine these densities, we resort to the thermal occupation of each single-electron KS orbital $f_{\mu}$ at a given temperature $T$ and chemical potential $\mu$,

$$
f_{\mu}\left(\varepsilon_{n k}\right)=\frac{1}{1+e^{\left(\varepsilon_{n k}-\mu\right) / k_{B} T}} .
$$

This Fermi function gives the occupation of the $(n, k)$ state. The electron density is

$$
\begin{aligned}
\rho(y) & =\sum_{n} \frac{L}{2 \pi} \int d k\left\langle\Psi_{n k}\left|\delta\left(\mathbf{r}_{i}-\mathbf{r}\right)\right| \Psi_{n k}\right\rangle_{\mathbf{r}_{t}} f_{\mu}\left(\varepsilon_{n k}\right) \\
& =\sum_{n} \frac{1}{2 \pi} \int d k\left[\left|\varphi_{n k}(y, \uparrow)\right|^{2}+\left|\varphi_{n k}(y, \downarrow)\right|^{2}\right] f_{\mu}\left(\varepsilon_{n k}\right),
\end{aligned}
$$

and the one-dimensional (1D) electron density along the $\mathrm{QW}$ is the integral of $\rho(y)$ over $y$,

$$
\rho_{1 D}=\int d y \rho(y) .
$$

Translational invariance along the wire implies that all densities - actually all physical variables—only depend on $y$.

For the $a=x, y, z$ components of the spin magnetization, we have in a similar way

$$
m_{a}(y)=\sum_{n} \frac{L}{2 \pi} \int d k\left\langle\Psi_{n k}\left|\delta\left(\mathbf{r}_{i}-\mathbf{r}\right) \sigma_{a}\right| \Psi_{n k}\right\rangle_{i} f_{\mu}\left(\varepsilon_{n k}\right),
$$

where $\sigma_{a}$ is the corresponding Pauli matrix. The three components then read

$$
\begin{aligned}
& m_{x}(y)=\sum_{n} \frac{1}{2 \pi} \int d k 2 \operatorname{Re}\left[\varphi_{n k}(y, \uparrow)^{*} \varphi_{n k}(y, \downarrow)\right] f_{\mu}\left(\varepsilon_{n k}\right), \\
& m_{y}(y)=\sum_{n} \frac{1}{2 \pi} \int d k 2 \operatorname{Im}\left[\varphi_{n k}(y, \uparrow)^{*} \varphi_{n k}(y, \downarrow)\right] f_{\mu}\left(\varepsilon_{n k}\right), \\
& m_{z}(y)=\sum_{n} \frac{1}{2 \pi} \int d k\left[\left|\varphi_{n k}(y, \uparrow)\right|^{2}-\left|\varphi_{n k}(y, \downarrow)\right|^{2}\right] f_{\mu}\left(\varepsilon_{n k}\right) .
\end{aligned}
$$

To carry out the $k$ integrations, we have discretized the integrals in a $\left[-k_{\max },+k_{\max }\right]$ interval and have computed $\Psi_{n k}$ for the chosen states on a $k$ grid with $N_{k}$ points for all the $n$ 's up to a chosen $n_{\max }$. Next, we have performed the integrations using a high precision method-a Bode rule-in the $k$ domain. ${ }^{10}$ This discretization in $k$ space has implications in the way we handle the KS Hamiltonian we describe next.

\section{B. Kohn-Sham Hamiltonian}

We split the Kohn-Sham Hamiltonian $h_{K S}[\rho, \mathbf{m}]$ into three different pieces, $h_{K S}=h_{0}+h_{S O}+h_{Z}$, consisting of the kinetic plus confining and exchange-correlation (XC) terms, the Rashba plus Dresselhaus SO term, and the Zeeman contribution arising from an in-plane magnetic field applied with an arbitrary orientation 


$$
\mathbf{B}=B\left(\cos \phi_{B} \mathbf{u}_{x}+\sin \phi_{B} \mathbf{u}_{y}\right),
$$

where $\phi_{B}$ is the azimuthal angle. The extension to include a vertical magnetic field can be done easily, but it is not addressed here. Explicitly,

$$
\begin{gathered}
h_{0}=\frac{p_{x}^{2}+p_{y}^{2}}{2 m}+\frac{1}{2} m \omega_{0}^{2} y^{2}+V_{\eta \eta^{\prime}}^{x c}(y), \\
h_{S O}=\frac{\lambda_{R}}{\hbar}\left(p_{y} \sigma_{x}-p_{x} \sigma_{y}\right)+\frac{\lambda_{D}}{\hbar}\left(p_{x} \sigma_{x}-p_{y} \sigma_{y}\right), \\
h_{Z}=\mathcal{E}_{z}\left(\sigma_{x} \cos \phi_{B}+\sigma_{y} \sin \phi_{B}\right) .
\end{gathered}
$$

Note that since $B$ is in-plane, $p_{x}$ and $p_{y}$ are the actual components of the linear momentum of the electron and not the generalized momentum components that include contributions from the vector potential $\mathbf{A}, \mathbf{B}=\nabla \times \mathbf{A}$. We have introduced the self-consistent potential $V_{\eta \eta^{\prime}}^{x c}(y)$ due to exchange and correlation energies, the Zeeman energy $\mathcal{E}_{Z}=g^{*} \mu_{B} B$, and the Rashba and Dresselhaus parameters $\lambda_{R}$ and $\lambda_{D}$. In principle, one should also include a Hartree term, ${ }^{8,11}$ but we con- sider that it is exactly cancelled by some neutralizing background contribution-full screening approximation. ${ }^{12}$ The inclusion of such a Hartree term would introduce some uncertainties in the model, as its actual expression would depend on the way the positive charges are distributed to cancel out the divergence in the Hartree potential. To be definite, the screened transverse potential is assumed to be of parabolic type although other potentials such as a square well would yield a qualitatively similar behavior.

In view of the form of the Rashba and Dresselhaus contributions, it is quite natural to introduce a complex SO coupling parameter $\gamma \equiv \lambda_{D}+i \lambda_{R}$. Due to the translational invariance in the $x$ direction, the full spin-orbit contribution then reads

$$
h_{R}+h_{D}=\left(\begin{array}{cc}
0 & \gamma k+\gamma^{*} \frac{d}{d y} \\
\gamma^{*} k-\gamma \frac{d}{d y} & 0
\end{array}\right) \text {, }
$$

and from Eq. (2) we obtain the eigenvalue equation

$$
\left(\begin{array}{cc}
-\frac{\hbar^{2}}{2 m} \frac{d^{2}}{d y^{2}}+\frac{\hbar^{2} k^{2}}{2 m}+\frac{1}{2} m \omega_{0}^{2} y^{2}+V_{\uparrow \uparrow}^{x c} & \gamma^{*} \frac{d}{d y}+\gamma k+\mathcal{E}_{Z} e^{-i \phi_{B}}+V_{\uparrow \downarrow}^{x c} \\
-\gamma \frac{d}{d y}+\gamma^{*} k+\mathcal{E}_{Z} e^{i \phi_{B}}+V_{\downarrow \uparrow}^{x c} & -\frac{\hbar^{2}}{2 m} \frac{d^{2}}{d y^{2}}+\frac{\hbar^{2} k^{2}}{2 m}+\frac{1}{2} m \omega_{0}^{2} y^{2}+V_{\downarrow \downarrow}^{x c}
\end{array}\right)\left(\begin{array}{c}
\varphi_{n k \uparrow} \\
\varphi_{n k \downarrow}
\end{array}\right)=\varepsilon_{n k}\left(\begin{array}{c}
\varphi_{n k \uparrow} \\
\varphi_{n k \downarrow}
\end{array}\right),
$$

where $\varphi_{n k \eta} \equiv \varphi_{n k}(y, \eta)$. The Hermiticity of the above equation is apparent.

\section{Noncollinear local-spin density approximation}

The exchange-correlation potential matrix $V_{\eta \eta^{\prime}}^{x c}(y)$ is related to the energy functional $\mathcal{E}_{x c}$ for a uniformly polarized electron gas in the noncollinear LSDA framework. A useful presentation of the theory is given in Ref. 13; see also Ref. 14 for a recent application to semiconductor nanostructures. The underlying idea is that the local approximation is extended to locally treat the orientation of the magnetization exactly as in the uniformly magnetized system. To do so, one performs a local diagonalization of the density matrix, defining the angles $\theta(y)$ and $\phi(y)$ that give the orientation of the spin at point $y$-we recall that the system is translational invariant in the $x$ direction. In the noncollinear case, one deals with the density matrix

$$
\begin{aligned}
\rho_{\eta \eta^{\prime}}(y)= & \sum_{n} \frac{1}{2 \pi} \int d k \varphi_{n k}^{*}(y, \eta) \varphi_{n k}\left(y, \eta^{\prime}\right) f_{\mu}\left(\varepsilon_{n k}\right) \\
& \Rightarrow\left(\begin{array}{cc}
\rho_{\uparrow \uparrow} & \rho_{\uparrow \downarrow} \\
\rho_{\downarrow \uparrow} & \rho_{\downarrow \downarrow}
\end{array}\right)
\end{aligned}
$$

that is written in terms of the particle and magnetization densities, Eqs. (4) and (7),

$$
\left(\begin{array}{cc}
\rho_{\uparrow \uparrow} & \rho_{\uparrow \downarrow} \\
\rho_{\downarrow \uparrow} & \rho_{\downarrow \downarrow}
\end{array}\right)=\frac{1}{2}\left(\begin{array}{cc}
\rho+m_{z} & m_{x}+i m_{y} \\
m_{x}-i m_{y} & \rho-m_{z}
\end{array}\right) .
$$

Following Ref. 13, we define a diagonal density matrix by means of a local unitary transformation $U$,

$$
U \rho U^{+}=n \equiv\left(\begin{array}{cc}
n_{\uparrow} & 0 \\
0 & n_{\downarrow}
\end{array}\right) .
$$

The local rotation is given by

$$
U=\left(\begin{array}{cc}
e^{i \phi(y) / 2} \cos \frac{\theta(y)}{2} & e^{-i \phi(y) / 2} \sin \frac{\theta(y)}{2} \\
-e^{i \phi(y) / 2} \sin \frac{\theta(y)}{2} & e^{-i \phi(y) / 2} \cos \frac{\theta(y)}{2}
\end{array}\right),
$$

and the local rotation angles are determined by the equations

$$
\begin{gathered}
\tan \phi(y)=-\frac{m_{y}(y)}{m_{x}(y)}, \\
\tan \theta(y)=\frac{\sqrt{m_{x}^{2}(y)+m_{y}^{2}(y)}}{m_{z}(y)} .
\end{gathered}
$$

Omitting the $y$ arguments, the diagonal local densities are 


$$
\begin{aligned}
& n_{\uparrow}=\frac{1}{2}\left(\rho+m_{z} \cos \theta\right)+\operatorname{Re}\left\{\rho_{\uparrow \downarrow} e^{i \phi} \sin \theta\right\}, \\
& n_{\downarrow}=\frac{1}{2}\left(\rho-m_{z} \cos \theta\right)-\operatorname{Re}\left\{\rho_{\uparrow \downarrow} e^{i \phi} \sin \theta\right\} .
\end{aligned}
$$

Knowing $n_{\uparrow}$ and $n_{\downarrow}$ at a point $y$, we can use the familiar relations of collinear LSDA to compute the exchangecorrelation potentials

$$
\left(\begin{array}{cc}
v_{\uparrow} & 0 \\
0 & v_{\downarrow}
\end{array}\right) \equiv\left(\begin{array}{cc}
\delta \mathcal{E}_{x c}\left[n_{\uparrow}, n_{\downarrow}\right] / \delta n_{\uparrow} & 0 \\
0 & \delta \mathcal{E}_{x c}\left[n_{\uparrow}, n_{\downarrow}\right] / \delta n_{\downarrow}
\end{array}\right) .
$$

Finally, we undo the rotation. The resulting expression for the exchange-correlation potential $V^{x c}$ can be written as

$$
V_{\eta \eta^{\prime}}^{x c}(y) \equiv\left(\begin{array}{cc}
v_{0}+\Delta v \cos \theta & \Delta v e^{-i \phi} \sin \theta \\
\Delta v e^{i \phi} \sin \theta & v_{0}-\Delta v \cos \theta
\end{array}\right),
$$

where we have defined $v_{0} \equiv\left(v_{\uparrow}+v_{\downarrow}\right) / 2$ and $\Delta v \equiv\left(v_{\uparrow}-v_{\downarrow}\right) / 2$. This scheme fully determines the $2 \times 2$ potential matrix $V^{x c}$ in terms of the spinor orbitals and the LSDA energy density functional. It allows the description of spin textures where the spin orientation varies from one point to another, yielding a transverse profile for the spin orientation across the wire.

As in our previous works on two-dimensional nanoscopic structures, $\mathcal{E}_{x c}$ has been constructed from the results on the nonpolarized and fully polarized two-dimensional electron gas, ${ }^{15}$ using the two-dimensional von Barth and Hedin ${ }^{16}$ prescription to interpolate between both regimes. We want to mention that a more accurate expression for $\mathcal{E}_{x c}$ has become available. ${ }^{17}$ Its use would also render unnecessary the mentioned interpolation. While a very accurate description of the exchange-correlation energy is needed for a quantitative description of the Wigner crystallization at low electron densities and the corresponding phase diagram, we do not expect that the use of the "improved" $\mathcal{E}_{x c}$ might introduce substantial changes in the results we are going to discuss. We would also like to recall that in the presence of an external $B$, the exchange-correlation energy not only depends on $n_{\uparrow, \downarrow}$ but also on the paramagnetic currents, constituting the so-called current spin-density-functional theory (CSDFT), ${ }^{18}$ which is better suited to high magnetic fields than LSDA. CSDFT has been applied to study two-dimensional quantum dots and rings. ${ }^{19-22}$ We have carried out in the past some comparisons between the results obtained using LSDA and CSDFT, and the overall agreement turns out to be satisfactory, indicating that the effects caused by the inclusion of the paramagnetic currents are small, see, e.g., Ref. 23.

For each $(n k)$, we, therefore, have to diagonalize Eq. (2), keeping the lowest $n_{\max }$ eigenvalues and eigenvectors $\left\{\varepsilon_{n k}, \Psi_{n k}(\mathbf{r})\right\}$. To do so, we introduce a $y$ discretization from $-y_{\max }$ to $+y_{\max }$. This defines $N_{y}$ points, and since the two components are coupled, the resulting matrix is $2 N_{y} \times 2 N_{y}$. Once $\left\{\varepsilon_{n k}, \Psi_{n k}(\mathbf{r})\right\}$ are known, we proceed to compute the density and magnetization, which allow one to start a new iteration until self-consistency is achieved. For $N_{y}$ 's of the order of 100, the diagonalization is extremely fast and, although it is repeated $N_{k} \times n_{\max }$ times per iteration, the calculation is quite efficient.
For the present study, we have considered the case of a GaAs heterostructure and have used the corresponding bulk parameters, namely, $g^{*}=-0.44, m^{*}=0.067$, and dielectric constant $\epsilon=12.4$. The experimental values for the GaAs/AlGaAs SO coupling constants $\lambda_{R, D}$ are of the order of $10^{-11} \mathrm{eV} \mathrm{m} .^{24-26}$

Rather than presenting an exhaustive study as a function of the parameter space of the model, we have limited ourselves to some illustrative examples. In particular, when different from zero, the magnetic field has been set to $B$ $=20 \mathrm{~T}$, and only two angles have been considered, namely, $\phi_{B}=0$ and $\pi / 2$. To present the results, we have used the harmonic oscillator length $l_{0}=\sqrt{\hbar / m \omega_{0}}$ to express both the linear density $\rho_{1 D}$ and the wave number $k$ in units of $l_{0}^{-1}$. For a typical energy value $\hbar \omega_{0}=4 \mathrm{meV}$, a unit linear density $\rho_{1 D}=l_{0}^{-1}$ is about $5.9 \times 10^{5} \mathrm{~cm}^{-1}$. The interaction-toconfinement ratio $e^{2} /\left(\epsilon l_{0} \hbar \omega_{0}\right)$ has been fixed to 1.72 , corresponding to the above typical values for GaAs and to $\hbar \omega_{0}$ $=4 \mathrm{meV}$. The energies are expressed in $\hbar \omega_{0}$ units.

To characterize the SO regime, we have used the ratio of the SO to the confining energy, namely,

$$
\Delta_{R, D}=\frac{m \lambda_{R, D}^{2}}{2 \hbar^{3} \omega_{0}}
$$

We have used the values $\Delta_{R}=0.0037$ and $\Delta_{D}=0.015$ to represent a typical weak SO coupling regime, and the values $\Delta_{R}=0.093$ and $\Delta_{D}=0.37$ to represent a typical strong SO coupling regime. The weak and strong coupling results we discuss in the following have been obtained using these parameters, except when their values are explicitly given.

We have chosen the linear electronic density $\rho_{1 D}$ as the natural variable to characterize the $\mathrm{QW}^{8}$ and have solved the KS equations to determine the corresponding subband structure and chemical potential $\mu$. Contrary to the noninteracting situation, in which for a given set of physical parameters defining the QW, its subband structure is determined once for all and can be filled with electrons to reach a prefixed $\rho_{1 D}$ or $\mu$ value, in the present case, the subband structure has to be self-consistency determined and may change from a linear density to another.

At a given $\rho_{1 D}$, the energy per unit length

$$
\begin{aligned}
E_{\text {total }} & =E_{k i n}+E_{\text {conf }}+E_{S O}+E_{x c}+E_{Z} \\
& =\int d y \sum_{n} \frac{L}{2 \pi} \int d k\left\langle\Psi_{n k}\left|h_{k s}\right| \Psi_{n k}\right\rangle
\end{aligned}
$$

is calculated piece by piece. For the kinetic and confining terms, we obtain

$$
\begin{aligned}
E_{k i n}= & \frac{1}{4 \pi} \int d y \sum_{n} \int d k\left\{\left|\varphi_{n k \uparrow}^{\prime}\right|^{2}+\left|\varphi_{n k \downarrow}^{\prime}\right|^{2}+k^{2}\left(\left|\varphi_{n k \uparrow}\right|^{2}\right.\right. \\
& \left.\left.+\left|\varphi_{n k \downarrow}\right|^{2}\right)\right\} f_{\mu}\left(\varepsilon_{n k}\right),
\end{aligned}
$$




$$
E_{c o n f}=\frac{\omega_{0}^{2}}{4 \pi} \int d y \sum_{n} \int d k y^{2}\left(\left|\varphi_{n k \uparrow}\right|^{2}+\left|\varphi_{n k \downarrow}\right|^{2}\right) f_{\mu}\left(\varepsilon_{n k}\right),
$$

with $\varphi_{n k \eta}^{\prime} \equiv d \varphi_{n k \eta} / d y$. For the SO contribution, using the expression of $h_{R}$ and $h_{D}$ in terms of $\gamma$, we have

$$
\begin{aligned}
& E_{S O}=\int d y \sum_{n} \frac{1}{2 \pi} \int d k\left(\varphi_{n k \uparrow}^{*} \varphi_{n k \downarrow}^{*}\right) \\
& \times\left(\begin{array}{cc}
0 & \gamma k+\gamma^{*} \frac{d}{d y} \\
\gamma^{*} k-\gamma \frac{d}{d y} & 0
\end{array}\right)\left(\begin{array}{l}
\varphi_{n k \uparrow} \\
\varphi_{n k \downarrow}
\end{array}\right) f_{\mu}\left(\varepsilon_{n k}\right) .
\end{aligned}
$$

Performing the matrix multiplications, we get an expression that is apparently real

$$
\begin{aligned}
E_{S O}= & \int d y \sum_{n} \frac{1}{2 \pi} \int d k\left\{2 k \mathcal{R} e\left[\gamma^{*} \varphi_{n k \downarrow}^{*} \varphi_{n k \uparrow}\right]\right. \\
& \left.+\operatorname{Re} e\left[\gamma\left(\varphi_{n k \downarrow}^{\prime *} \varphi_{n k \uparrow}-\varphi_{n k \downarrow}^{*} \varphi_{n k \uparrow}^{\prime}\right)\right]\right\} f_{\mu}\left(\varepsilon_{n k}\right) .
\end{aligned}
$$

To obtain the above expression, we have used

$$
\int d y \varphi_{n k \uparrow}^{*} \varphi_{n k \downarrow}^{\prime}=\frac{1}{2} \int d y\left(\varphi_{n k \uparrow}^{*} \varphi_{n k \downarrow}^{\prime}-\varphi_{n k \uparrow}^{\prime *} \varphi_{n k \downarrow}\right) .
$$

Finally, we have the Zeeman and exchange-correlation contributions,

$$
\begin{gathered}
E_{Z}=\mathcal{E}_{Z} \int d y \sum_{n} \frac{1}{2 \pi} \int d k 2\left\{\cos \phi_{B} \mathcal{R} e\left[\varphi_{n k \downarrow}^{*} \varphi_{n k \uparrow}\right]\right. \\
\left.-\sin \phi_{B} \mathcal{I} m\left[\varphi_{n k \downarrow}^{*} \varphi_{n k \uparrow}\right]\right\} f_{\mu}\left(\varepsilon_{n k}\right), \\
E_{x c}=\int d y \varepsilon_{x c}(y) \rho(y),
\end{gathered}
$$

where $\varepsilon_{x c}(y)$ is the exchange-correlation energy per electron.

We have also computed the total energy in an alternative way that explicitly uses the eigenvalues obtained, solving the KS equations, see, e.g., Sec. 4.4 of Ref. 27:

$$
\begin{aligned}
E_{\text {total }}= & \sum_{n} \frac{1}{2 \pi} \int d k \varepsilon_{n k} f_{\mu}\left(\varepsilon_{n k}\right)+\int d y \varepsilon_{x c}(y) \rho(y) \\
& -\int d y\left(n_{\uparrow} v_{\uparrow}+n_{\downarrow} v_{\downarrow}\right) .
\end{aligned}
$$

We have checked that it gives the same result as Eq. (21) with a high accuracy, constituting a stringent test on our numerical method.

Figure 1 shows the energy per electron $E / N$ at $B=0$ as a function of $\rho_{1 D}$ in the weak and strong SO coupling regimes. Due to the exchange-correlation energy, ${ }^{8} E / N$ is not a monotonous function of the linear density, and neither is the chemical potential $\mu$.

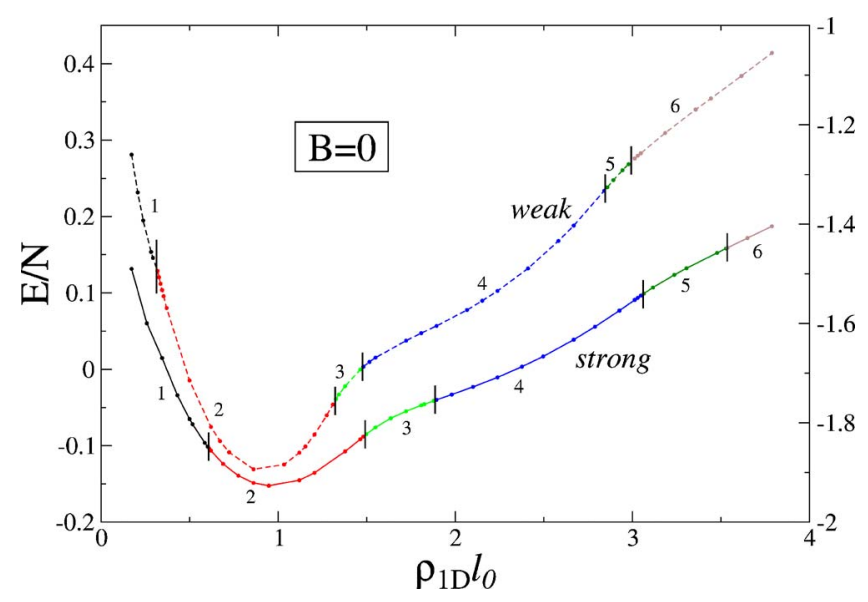

FIG. 1. (Color online) Energy per electron (in $\hbar \omega_{0}$ units) as a function of the linear density at $B=0$. The regions separated by vertical lines are characterized by the indicated number of distinct subbands crossed by the electron chemical potential $\mu$, i.e., partially occupied subbands. Some of these subbands are crossed more than twice by $\mu$, producing the anomalous steps in the conductance discussed in Sec. III. The vertical left (right) scale corresponds to the weak (strong) SO regime. The lines have been drawn to guide the eye.

\section{Subband structure}

We have studied the effect of the exchange-correlation interaction in several situations involving in-plane magnetic fields and different strengths of the SO interaction, and have found it difficult to systematize, as its effect depends on the actual value of other variables that characterize the QW, such as $\rho_{1 D}$, the orientation $\phi_{B}$ of the applied $B$, and the values of the SO coupling constants. In general, $V^{x c}$ has a tendency to enhance magnetic field effects, and-perhaps the most interesting feature- to act in some cases as an applied magnetic field, especially at low densities. Indeed, we have found that for some configurations, the subband structure at $B=0$, when $V^{x c}$ is taken into account, turns out to be qualitatively the same as when a certain $B$ is applied and $V^{x c}=0$. The spontaneous symmetry breaking leading to the appearance of a magnetization is made possible by the exchange-correlation energy. In some cases, this term attains its minimal value when the system polarizes itself, even though no magnetic field is present. Therefore, one can physically ascribe the resulting magnetization to an effective magnetic field originating in the quantum exchange-correlation energy. Analogously, when a magnetic field is already applied, $V^{x c}$ may act as if it were an additional field increasing the value of the actual $B$ field, or contributing to create an effective in-plane magnetic field with an orientation different from that of the applied field. Likely, the lack of a common spin axis when SO effects are taken into account has much to do with the complex effect of $V^{x c}$ on the subband structure.

Since in many previous works only the Rashba SO interaction has been taken into account, it is pertinent to begin with the discussion of the $V^{x c}$ effects in the $\Delta_{D}=0$ situation. As an example, Fig. 2 shows the results corresponding to a low density QW, $\rho_{1 D} l_{0}=0.17$, for an applied $B$ field of $20 \mathrm{~T}$ 


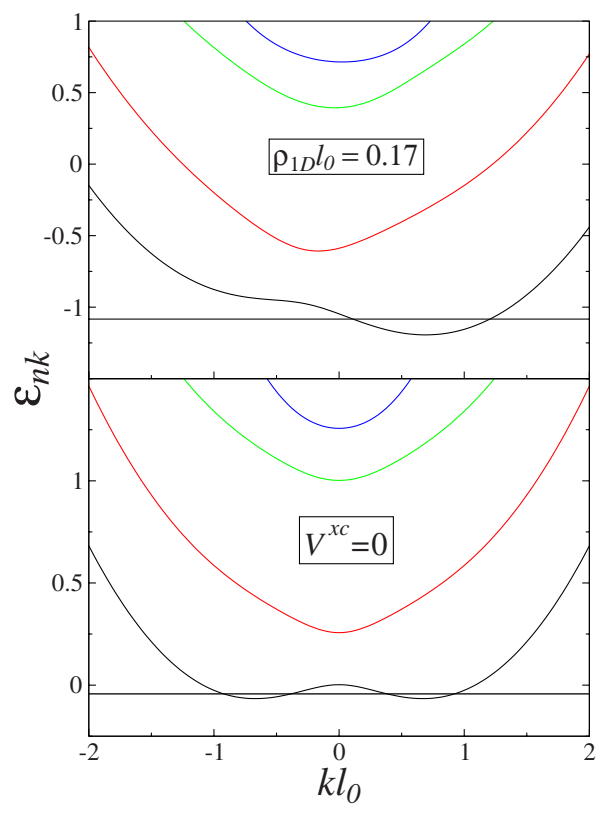

FIG. 2. (Color online) Single-electron energies (in $\hbar \omega_{0}$ units) for $\rho_{1 D} l_{0}=0.17$ and $B=20 \mathrm{~T}, \phi_{B}=0$, in a strong SO regime characterized by $\Delta_{R}=0.37$ and $\Delta_{D}=0$, as a function of the linear momentum $k l_{0}$. The thin horizontal line represents the chemical potential. The effect of $V^{x c}$ has not been included in the results shown in the bottom panel.

and $\phi_{B}=0$ in a strong SO regime, namely, $\Delta_{R}=0.37$. One may see that when $V^{x c}=0$, the first subband presents the symmetric double minimum structure already found by other authors, ${ }^{4,9}$ whose existence yields anomalous steps in the conductance; see, e.g., Fig. 2(c) of Ref. 9 and Fig. 5, top panel, of Ref. 4. The effect of $V^{x c}$ at this low density is to induce an asymmetry in the lowest subbands, transforming the symmetric double minimum structure, characteristic of the $V^{x c}=0, \phi_{B}=0$ case when only the Rashba term is considered, into a structure rather similar to the one corresponding to $V^{x c}=0$ and $\phi_{B}=\pi / 4$, as shown in Fig. 2(b) of Ref. 9. This effect yields only one minimum below the chemical potential contributing to the conductance $G$; see the next section. We want to point out that, for small $\Delta_{R}$ values, the double minimum structure is not found even when $V^{x c}=0$, whereas in a very strong regime, e.g., $\Delta_{R}=0.83$, this structure is also present for odd $n>1$ values. In this case, the changes induced by $V^{x c}$ are qualitatively similar to those displayed in Fig. 2(b).

When the SO interaction is included, spin is not a good quantum number and it is possible to find spin textures across the wire. ${ }^{4}$ This is illustrated in Fig. 3, which corresponds to the situation displayed in Fig. 2. The left panel corresponds to the $V^{x c}=0$ case. The vector plot shows the in-plane spin magnetization, and the solid line corresponds to the $z$ component [see Eq. (7)]. In both panels, the $z$ component displays spin accumulations of different sign on opposite sides of the wire, ${ }^{3,4}$ indicating the robustness of this effect with $V^{x c}$. On the contrary, the in-plane spin distributions show remarkable differences. When $V^{x c}=0$, it is perfectly aligned in the direction of the magnetic field $\left(\phi_{B}=0\right)$, while

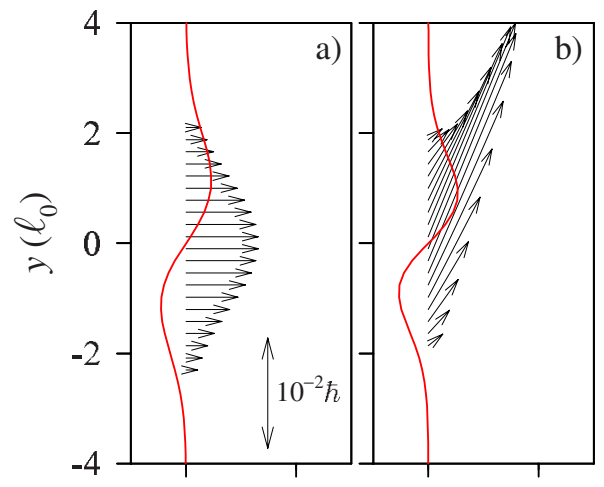

FIG. 3. (Color online) Spin textures across the wire ( $y$ direction, in $l_{0}$ units) corresponding to the situation displayed in Fig. 2. The left panel corresponds to the $V^{x c}=0$ case. The vector plot shows the in-plane spin, and the solid line corresponds to the $z$ component. The spin scale is indicated in (a).

it deviates, pointing in a tilted orientation with $\phi_{B} \approx \pi / 4$, when $V^{x c}$ is included. This result nicely illustrates the property mentioned before that $V^{x c}$ amounts to replace the applied magnetic field with an effective one having different modulus and direction.

Exchange-correlation effects also appear when both SO contributions are taken into account. Figure 4 shows the energy subband structure in one of the most interesting situations for the discussion of the conductance that we will carry out in the next section. It corresponds to the strong coupling regime for a $\phi_{B}=\pi / 2$ magnetic field. In both panels, conspicuous subband gaps and local extrema appear near $k=0$. The interesting feature is the weak local maximum at $k>0$ for the even subbands when $V^{x c}$ is not considered (bottom panel). Similar structures have been found by Moroz and Barnes, ${ }^{1}$ who address the $B=0$ case for the Rashba SO interaction. The existence of these maxima is the reason for the "anomalous steps" in the conductance that appear on top of the "ordinary steps" at even $e^{2} / h$ values. The inclusion of $V^{x c}$ washes out these structures, as can be seen in the top panel of Fig. 4. On the other hand, the well-known ${ }^{9,4}$ local extrema present in the odd bands, responsible for the anomalous structures on top of the ordinary steps corresponding to odd $e^{2} / h$ values, remain qualitatively unaffected by the inclusion of the exchange-correlation interaction. Another situation is shown in Fig. 5, again in the strong SO regime, corresponding to $B=0$ and $\rho_{1 D} l_{0}=0.52$. It can be seen that $V^{x c}$ produces a subband structure similar to that of the bottom panel of Fig. 2 and this is also reflected in the conductance, as will be discussed in the next section.

The spin textures corresponding to the results including $V^{x c}$ of Figs. 4 and 5 are shown in the left and right panels of Fig. 6, respectively. The left-panel results correspond to a magnetic field along $+y$ which is clearly constraining the in-plane magnetization to essentially point along this direction. However, some straggling of the arrows around the vertical direction persists. As in Fig. 3, the $z$ component displays different sign accumulations on opposite edges of the wire that, when combined with the in-plane distribution, yield a rather complicated spin texturing. The right panel in Fig. 6 


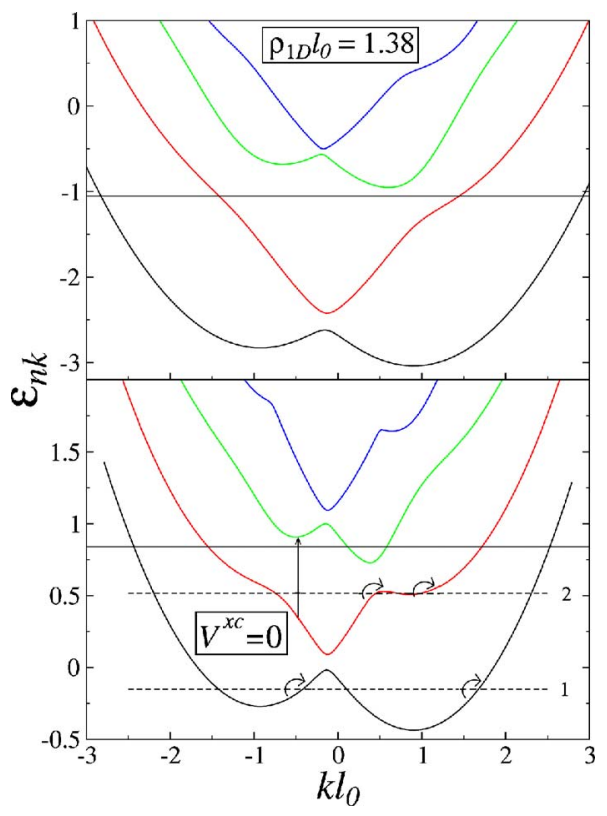

FIG. 4. (Color online) Single-electron energies (in $\hbar \omega_{0}$ units) for $\rho_{1 D} l_{0}=1.38$ and $B=20 \mathrm{~T}, \phi_{B}=\pi / 2$, in a strong SO regime characterized by $\Delta_{R}=0.093$ and $\Delta_{D}=0.37$, as a function of the linear momentum $k l_{0}$. $V^{x c}$ has not been included in the results shown in the bottom panel. In both panels, the thin horizontal line represents the chemical potential for the linear density $\rho_{1 D} l_{0}=1.38$, whereas the dashed horizontal lines in the bottom panel represent the chemical potential for two smaller linear densities chosen to show different kinds of intrasubband excitations, represented by curved arrows near the corresponding Fermi level, that contribute to the QW conductance, as discussed in Sec. III. The vertical arrow in the bottom panel represents an intersubband transition.

corresponds to a $B=0$ case. In this situation, there is no preferred direction a priori and, therefore, the fact that $V^{x c}$ may induce a spin texture like that in Fig. 6(b), where the in-plane spin selects a certain direction, is an example of spontaneous symmetry breaking induced by the interaction.

A particular situation - see the Appendix - appears when $\lambda_{D}=\lambda_{R}$ at zero magnetic field. It has already been addressed when $V^{x c}=0,{ }^{28}$ showing that subband anticrossings disappear when both SO strengths are equal. We have found that the inclusion of $V^{x c}$ does not change the crossing properties of the subbands in any SO regime, and that it only induces a small subband splitting.

Exchange-correlation effects are also found in the weak SO coupling regime, though in this case no local extrema appear. However, we will see in Sec. III that the effects of $V^{x c}$ on the conductance are also visible. As said above, all these $V^{x c}$ features are especially marked at low densities, becoming notably weaker or disappearing for $n \geqslant 2$, in which case, only small $k$ asymmetries are observed in odd subbands when $B=0$. When an in-plane $B$ acts on the $\mathrm{QW}$ in the weak SO coupling regime, the most apparent effect of $V^{x c}$ is to slightly enhance the $B$ effects without producing qualitative changes in the subband structure.

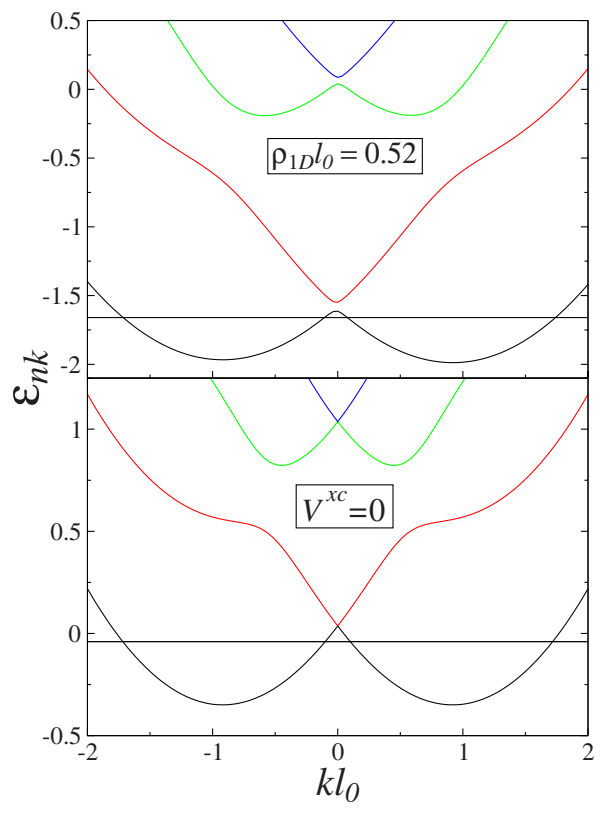

FIG. 5. (Color online) Single-electron energies (in $\hbar \omega_{0}$ units) for $\rho_{1 D} l_{0}=0.52$ and $B=0 \mathrm{~T}$ in a strong $\mathrm{SO}$ regime characterized by $\Delta_{R}=0.093$ and $\Delta_{D}=0.37$, as a function of the linear momentum $k l_{0}$. The thin horizontal line represents the chemical potential. $V^{x c}$ has not been included in the results shown in the bottom panel.

\section{CONDUCTANCE OF QUANTUM WIRES}

The noncollinear Kohn-Sham calculation discussed in the previous section allows one to evaluate the KS-mean fieldlinear density response $\chi_{0}(q, \omega)$ to a field parallel to the wire, i.e., in the $x$ direction, which involves only intrasubband excitations:

$$
\frac{\chi_{0}(q, \omega)}{L}=\frac{1}{\pi} \sum \int d k \frac{\epsilon_{n k, q}\left|\left\langle\Psi_{n k+q}\left|e^{i q x}\right| \Psi_{n k}\right\rangle\right|^{2}}{(\omega+i \lambda)^{2}-\epsilon_{n k, q}^{2}},
$$

where $\lambda$ is a small real quantity and the sum runs over all the possible intrasubband excitations of energy $\epsilon_{n k, q}$ induced

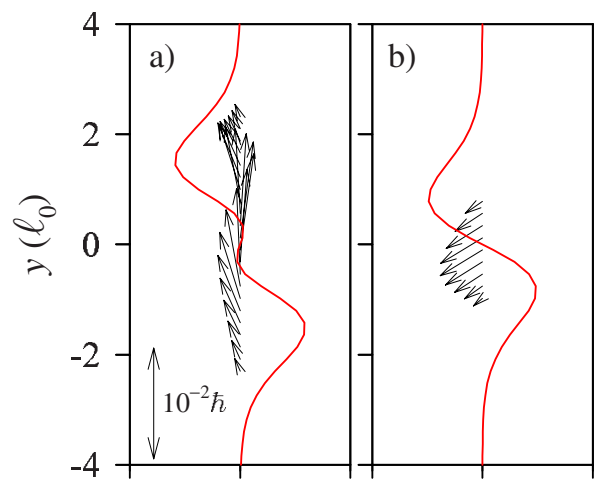

FIG. 6. (Color online) Spin textures across the wire ( $y$ direction, in $l_{0}$ units) corresponding to the situations displayed in Fig. 4 (left panel) and Fig. 5 (right panel) for the $V^{x c} \neq 0$ cases. The vector plot shows the in-plane spin, and the solid line corresponds to the $z$ component. The spin scale is indicated in (a). 
from the ground state by the density operator $\sum_{j} e^{i q x_{j}}$. The longitudinal conductivity associated with $\frac{\chi_{0}(q, \omega)}{L}$ is given by $^{27,29}$

$$
\frac{\sigma(\omega)}{L}=i \frac{e^{2} \omega}{q^{2}} \frac{\chi_{0}(q, \omega)}{L},
$$

whose real part is

$$
\operatorname{Re}\left[\frac{\sigma(\omega)}{L}\right]=-\frac{e^{2} \omega}{q^{2}} \operatorname{Im} \frac{\chi_{0}(q, \omega)}{L} .
$$

The conductance $G$ is defined as the $q \rightarrow 0, \omega \rightarrow 0$ limit of the above expression. There are two possible kinds of electronhole excitations for the subband patterns generated before, namely, intersubband (between different subbands) and intrasubband (inside a subband). The intrasubband excitations exist due to the $y$ confinement, which breaks the translational invariance of the system in the $y$ direction and bends the single-particle energy subbands. These excitations are gapless and are responsible for the $x x$ dc conductivity of the wire.

In the $q \rightarrow 0$ limit, the operator $\sum_{j} e^{i q x_{j}}$ induces intrasubband excitations between the states $\left|\Psi_{n k}\right\rangle$ and $\left|\Psi_{n k+q}\right\rangle$, with excitation energies given by

$$
\epsilon_{n k, q}=\epsilon_{n k+q}-\epsilon_{n k}=\left.q \frac{\partial \epsilon_{n k}}{\partial k}\right|_{k=k_{n}} \equiv \alpha_{k_{n}} q,
$$

where $k_{n}$ are the intersections of the $n$ subband with the chemical potential $\mu$ corresponding to positive slopes $\alpha_{k_{n}}$. Indeed, it is crucial to realize that for $q>0$, only intrasubband excitations with $k+q>k$ are allowed. Referring to Fig. 4 , these allowed transitions are represented as curved arrows for two possible situations that correspond to two different values of the chemical potential. Since

$$
\begin{aligned}
& \left\langle\Psi_{n k+q}\left|e^{i q x}\right| \Psi_{n k}\right\rangle \\
& \quad=\int d \mathbf{r} \Psi_{n k+q}^{\dagger}(\mathbf{r}) e^{i q x} \Psi_{n k}(\mathbf{r}) \\
& \quad=\frac{1}{L} \sum_{\eta} \iint d x d y \varphi_{n k+q}^{*}(y, \eta) e^{-i(k+q) x} e^{i q x} \varphi_{n k}(y, \eta) e^{i k x} \\
& \quad=1+O(q)
\end{aligned}
$$

in the $q \rightarrow 0$ limit, the matrix elements of the operator $e^{i q x}$ can be taken equal to unity. In the same limit, the phase space for electron-hole excitations is $\int d k=q,{ }^{27}$ yielding

$$
\operatorname{Re}\left[\frac{\sigma(q, \omega)}{L}\right]=\frac{\pi e^{2}}{q^{2}} \sum_{k_{n}} \frac{q}{2 \pi} \alpha_{k_{n}} q \delta\left(\omega-\alpha_{k_{n}} q\right),
$$

where we have denoted with $\Sigma_{k_{n}}$ the sum over all the possible intrasubband allowed excitations. This amounts to counting the number of cuts of the chemical potential with partially occupied subbands corresponding to positive slope values $\alpha_{k_{n}}$. Taking the cosine Fourier transform, we arrive at

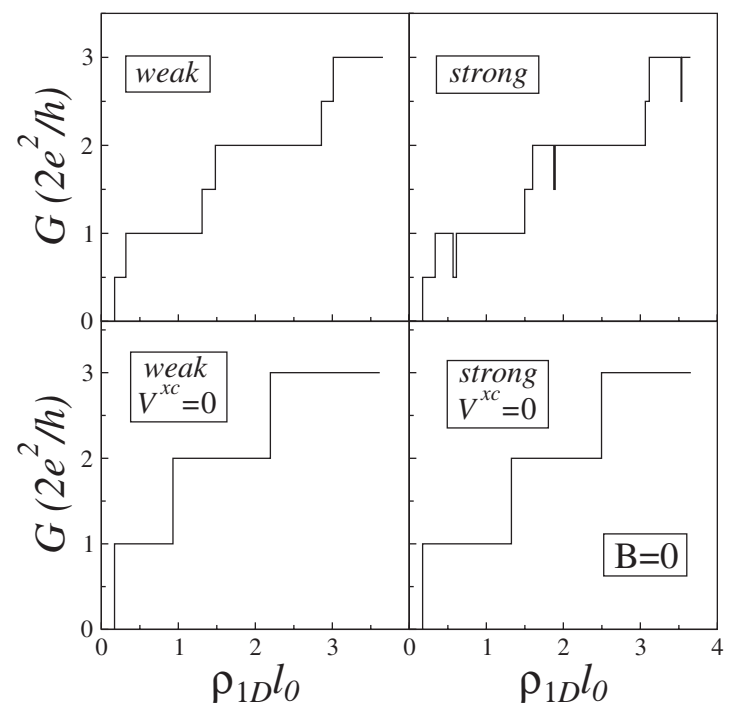

FIG. 7. Conductance as a function of the linear density for $B$ $=0$ in the strong and weak SO regimes when the exchangecorrelation energy is taken into account (top panels) and when it is not (bottom panels).

$$
\operatorname{Re}\left[\frac{\sigma(y, \omega)}{L}\right]=\frac{e^{2}}{2 \pi} \sum_{k_{n}} \cos \left(\frac{\omega y}{\alpha_{k_{n}}}\right) .
$$

Thus, in the $\omega \rightarrow 0$ limit, we obtain the conductance as

$$
G=\frac{e^{2}}{h} \sum_{k_{n}} 1 .
$$

In the $V^{x c}=0$ case, in the absence of $B$ and $\mathrm{SO}$ effects, $\epsilon_{n k}=(n+1 / 2) \omega_{0}+k^{2} / 2$ and the subbands are spin degenerate. Thus, only one intrasubband excitation (one single intersection $k_{n}$ ) contributes to $G$ for each subband $n$. As a consequence, Eq. (37) gives the usual conductance quantization of the Landauer formalism, where each spin degenerate subband contributes $e^{2} / h$ to the conductance, yielding the result $G=\frac{2 e^{2}}{h} \Sigma_{n} 1$. However, different results for $G$ may arise due to magnetic field and spin-orbit and $V^{x c}$ effects on the energy spectrum. This is illustrated in Figs. 7-9, which constitute the main result of this paper. In them, the conductance is expressed in $G_{0}=2 e^{2} / h$ units as a function of the linear electronic density.

Figure 7 shows the $B=0$ case for the weak and strong SO regimes when the exchange-correlation energy is taken into account and when it is not. It can be seen that when $V^{x c}=0$, the conductance displays the usual steps of the spin degenerate case commented before. Contrarily, when $V^{x c} \neq 0$, the induced spin splitting in the energy subbands gives rise to steps at semi-integer multiples of $G_{0}$ for both SO coupling regimes. These steps are apparently narrower than those corresponding to integer multiples of $G_{0}$ because the splitting of the subbands due to the confinement-coming from the $(n$ $+1 / 2) \omega_{0}$ term-is much larger than the one induced by $V^{x c}$.

An interesting feature appears in the strong SO regime, in which "anomalous plateaus" are found on top of the mentioned steps corresponding to semi-integer multiples of $G_{0}$. 


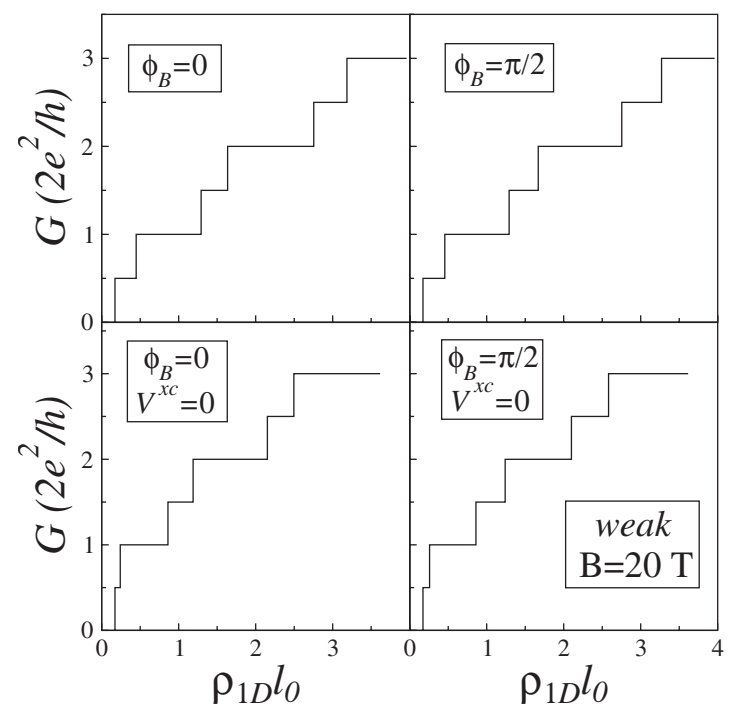

FIG. 8. Conductance as a function of the linear density for $B$ $=20 \mathrm{~T}$ in the weak SO regime when the exchange-correlation energy is taken into account (top panels) and when it is not (bottom panels). The azimuthal angle of the magnetic field is indicated.

Indeed, it can be seen that $G$ has a nonmonotonic behavior as a function of $\rho_{1 D} l_{0}$, but presents $0.5 G_{0}$ drops for some values of the electronic density. The origin of these plateaus can be inferred from the top panel of Fig. 5, in which the combination of the spin splitting induced by $V^{x c}$ and the well-known subband $k$ splitting induced by the (strong) SO coupling gives rise to two possible intrasubband excitations per subband (two intersection points $k_{n}$ ) in some small ranges of the chemical potential values (and thus of electronic densities), yielding the anomalous structure in the conductance. These anomalous structures have already been found when considering an applied in-plane magnetic field. ${ }^{4,9}$ Contrarily, in our case $B$ is zero, and thus, it is a genuine exchange-correlation interaction effect, which, as indicated in the previous section, seems to mimic in some cases the effect of an applied magnetic field.

Figure 8 shows the conductance in the weak SO regime when the magnetic field is applied along the $\phi_{B}=0$ and $\pi / 2$ directions. As expected, ${ }^{4,9}$ we have found plateaus at semiinteger multiples of $G_{0}$ even when $V^{x c}=0$. The larger effect of $V^{x c}$ at low densities can be inferred from the difference in the width of the first semi-integer steps when exchangecorrelation effects are included and when they are not, which shows that $V^{x c}$ combines with $B$, giving rise to a larger effective magnetic field. Some further evidence of the $B$-like acting of $V^{x c}$ stems from the comparison of the results of this figure with those displayed in the left panels of Fig. 7 corresponding to the $B=0$ weak $\mathrm{SO}$ coupling case.

Figure 9 shows the conductance in the strong SO regime. When $V^{x c} \neq 0$, the conductance is qualitatively similar to that at $B=0$ for the same SO regime-right top panel of Fig. 7 . Only the width of the anomalous plateaus varies appreciably, especially when $\phi_{B}=\pi / 2$. When $V^{x c}=0$ and $\phi_{B}=0$, the structure is similar to that displayed in the top panels of Fig. 9 but, as in the weak SO regime, the first semi-integer step is narrower than when $V^{x c} \neq 0$. As before, the same happens for

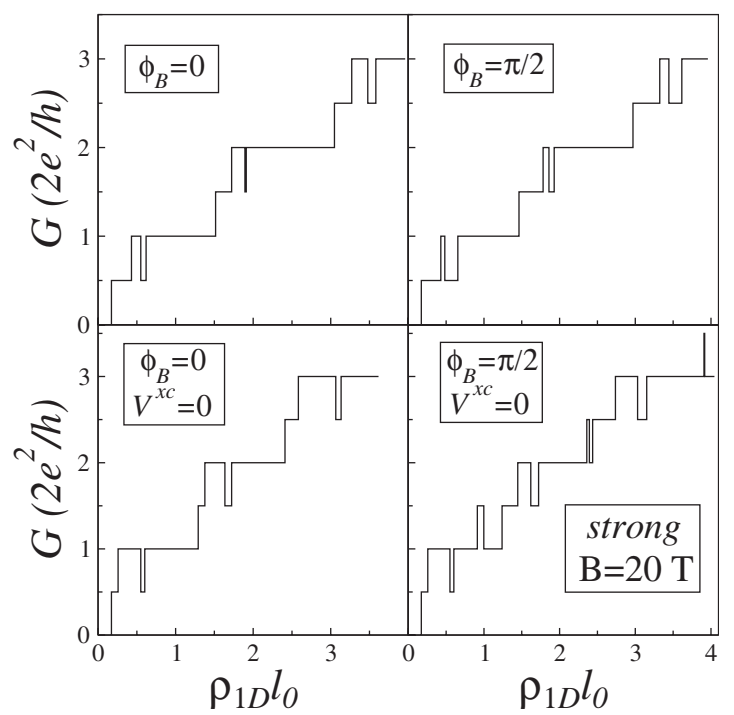

FIG. 9. Conductance as a function of the linear density for $B$ $=20 \mathrm{~T}$ in the strong SO regime when the exchange-correlation energy is taken into account (top panels) and when it is not (bottom panels). The azimuthal angle of the magnetic field is indicated.

most steps. New interesting structures appear when $\phi_{B}$ $=\pi / 2$ for the $V^{x c}=0$ case: in addition to the just mentioned ones at semi-integer multiples of $G_{0}$, anomalous plateaus at integer multiples of $G_{0}$ are also found. They are narrower than the semi-integer ones and their existence is due to the presence of local maxima in the subband spectrum for even values of $n$, which have already been discussed in Sec. II (see, e.g., the bottom panel of Fig. 4). It is worthy to note that these structures are not robust in the sense that the exchange-correlation energy washes them out (compare the right panels of Fig. 9). These additional anomalous plateaus have also been found in Ref. 1, where neither the $V^{x c}$ nor the Dresselhaus SO interactions were considered.

Finally, we want to point out that the above mentioned behavior is found when $\phi_{B}=0$ instead of $\pi / 2$, if the values of $\Delta_{R}$ and $\Delta_{D}$ are interchanged. This is due to the particular interplay of the Rashba and Dresselhaus SO interactions and the orientation of the magnetic field, which is discussed in the Appendix [see Eq. (A7)].

\section{SUMMARY}

Within the noncollinear LSDA and the linear response theory, we have studied the effect of the exchangecorrelation interaction on the ground state structure and conductance of quantum wires for different strengths of the Rashba and Dresselhaus spin-orbit interactions. We have also discussed the possibility to have an applied in-plane $B$ field.

We have found that in some cases, especially at low densities, $V^{x c}$ "combines" with the actual $B$ field or produces an effect similar as if it were an applied magnetic field. This manifests in the energy subband structure and in the QW conductance for all SO coupling strengths, although it is in the strong SO regime where the most striking features appear. In this case, we have found that the $V^{x c}$ induced spin 
splitting gives rise to the so-called anomalous plateaus at semi-integer multiples of $G_{0}$ even when $B=0$. Moreover, when the strong SO coupling is combined with the applied in-plane magnetic field, local maxima may appear in the even energy subbands, yielding anomalous plateaus also for integer multiples of $G_{0}$.

As a general trend, we have found that the effect of the exchange-correlation potential depends on the actual value of other variables defining the QW state, such as the applied magnetic field and/or the SO parameters considered in the calculation. The conductance turns out to be rather sensitive to the ground state structure of the QW. In particular, to symmetry breaking effects induced by $V^{x c}$, such as spontaneous spin polarization and Wigner crystallization, for which there seems to be some experimental evidence. ${ }^{30-32}$

\section{ACKNOWLEDGMENTS}

This work has been performed under Grants No. FIS2005-01414 and No. FIS2005-02796 from DGI (Spain), Grant No. 2005SGR00343 from Generalitat de Catalunya, and under Grant No. INFN07-30 from the Italian INFNSpanish DGI agreement.

\section{APPENDIX}

Even in the noninteracting case, the Hamiltonian Eq. (9) has no analytical solution except when $B=0$ and the Rashba and Dresselhaus SO terms have the same strength. ${ }^{28}$ In this case, the single-electron energies are

$$
\varepsilon_{n k}=\left(n+\frac{1}{2}\right) \hbar \omega_{0}+\frac{\hbar^{2}}{2 m}\left(k \pm \frac{\sqrt{2} m \lambda_{R}}{\hbar^{2}}\right)^{2}-\frac{2 \lambda_{R}^{2} m}{\hbar^{2}} .
$$

Besides the usual splitting of the subband structure, the effect of the SO interaction for this particular choice of the strength parameters is a rigid displacement of the subband structure. Remarkably, we have found that the inclusion of exchangecorrelation effects does not sensibly alter this independent particle result, irrespective of the SO regime-strong or weak.

The noninteracting case in the presence of an in-plane $B$ field has been worked out using second-order perturbation theory when only the Rashba term is present. ${ }^{4}$ We extend here these results considering both SO contributions. The noninteracting Hamiltonian can be written in dimensionless form as

$$
\begin{aligned}
\frac{H_{k}}{\hbar \omega_{0}}= & \left(\hat{n}_{k}+\frac{1}{2}\right)+\frac{1}{2}\left(\frac{l_{0}}{l_{Z}}\right)^{2}\left(\cos \phi_{B} \sigma_{x}+\sin \phi_{B} \sigma_{y}\right)+\frac{k^{2} l_{0}^{2}}{2} \\
& +\frac{k l_{0}^{2}}{2}\left(\tilde{l}_{D} \sigma_{x}-\tilde{l}_{R} \sigma_{y}\right)+\frac{i l_{0}}{2 \sqrt{2}}\left(\hat{a}_{k}^{\dagger}-\hat{a_{k}}\right)\left(\tilde{l}_{R} \sigma_{x}-\tilde{l}_{D} \sigma_{y}\right),
\end{aligned}
$$

where $\quad \tilde{l}_{R, D} \equiv l_{R, D}^{-1} \equiv 2 m \lambda_{R, D} / \hbar^{2}, \quad l_{0} \equiv \sqrt{\hbar / m \omega_{0}}, \quad$ and $\quad l_{Z}$ $\equiv \sqrt{\hbar^{2} / m g^{*} \mu_{B} B} \cdot \hat{a_{k}}{ }^{\dagger}$ and $\hat{a_{k}}$ appearing in this equation are the usual creation and annihilation harmonic oscillator operators

$$
{\hat{a_{k}}}^{\dagger}|n k \eta\rangle=\sqrt{n+1}|(n+1) k \eta\rangle,
$$

$$
\hat{a_{k}}|n k \eta\rangle=\sqrt{n}|(n-1) k \eta\rangle,
$$

where $\eta= \pm 1$. We split the Hamiltonian as $H_{k}=H_{k}^{0}+H_{k}^{S O}$ and consider the SO terms as a small perturbation. The other piece can be exactly solved, yielding the unperturbed energies - in $\hbar \omega_{0}$ units - and eigenvectors

$$
\begin{gathered}
E_{n k \eta}^{(0)}=\left(n+\frac{1}{2}\right)+\frac{k^{2} l_{0}^{2}}{2}+\frac{\eta}{2}\left(\frac{l_{0}}{l_{Z}}\right)^{2}, \\
|n k \eta\rangle=\frac{1}{\sqrt{2}} \varphi_{n}(y) e^{i k x}\left(\begin{array}{c}
1 \\
\eta e^{i \phi_{B}}
\end{array}\right) .
\end{gathered}
$$

We have calculated the first- and second-order energy corrections arising from

$$
\begin{aligned}
H_{k}^{S O} & =\frac{k l_{0}^{2}}{2}\left(\tilde{l}_{D} \sigma_{x}-\tilde{l}_{R} \sigma_{y}\right)+\frac{i l_{0}}{2 \sqrt{2}}\left(\hat{a}_{k}^{\dagger}-\hat{a_{k}}\right)\left(\tilde{l}_{R} \sigma_{x}-\tilde{l}_{D} \sigma_{y}\right) \\
& \equiv H_{k, 1}^{S O}+H_{k, 2}^{S O}
\end{aligned}
$$

The first-order correction is given by

$$
\begin{aligned}
& E_{n k \eta}^{(1)} \equiv\left\langle n k \eta\left|H_{k}^{S O}\right| n k \eta\right\rangle \\
& =\frac{k l_{0}^{2}}{2}\left(\frac{1}{\sqrt{2}}\right)^{2}\left(\begin{array}{ll}
1 & \eta e^{-i \phi_{B}}
\end{array}\right)\left(\begin{array}{cc}
0 & \tilde{l}_{D}+i \tilde{l}_{R} \\
\tilde{l}_{D}-i \tilde{l}_{R} & 0
\end{array}\right)\left(\begin{array}{c}
1 \\
\eta e^{i \phi_{B}}
\end{array}\right) \\
& =\frac{k l_{0}^{2}}{2} \eta\left(\tilde{l}_{D} \cos \phi_{B}-\tilde{l}_{R} \sin \phi_{B}\right) \text {. }
\end{aligned}
$$

As we can see, to first order only $H_{k, 1}^{S O}$ contributes, and setting $\lambda_{D}=0$, we recover the result of Ref. 4 . The first-order correction is zero when the magnetic field is oriented in the direction $\tan \phi_{B}=\lambda_{D} / \lambda_{R}$.

Interestingly, we see that to first order, the term that combines with the Zeeman splitting to yield multiple subband crossing when $\phi_{B}=0$ is the Rashba term, whereas it is the Dresselhaus term when $\phi_{B}=\pi / 2$, and have opposite signs. This result is not sensibly altered by exchange-correlation effects, and helps one to understand some of the detailed calculations shown in Sec. II. In particular, the conspicuous results that, for the same intensity of the SO interaction, the effect of the Dresselhaus term when $\phi_{B}=0$ is the same as that of the Rashba term at $\phi_{B}=\pi / 2$, provided $k$ is changed by $-k$.

Defining $|j\rangle \equiv|n k \eta\rangle$, the second-order correction is given by

$$
E_{j}^{(2)}=\sum_{i \neq j} \frac{\left|\left\langle i\left|H_{k}^{S O}\right| j\right\rangle\right|^{2}}{E_{j}^{(0)}-E_{i}^{(0)}}=\sum_{i \neq j} \frac{\left|\left\langle i\left|H_{k, 1}^{S O}+H_{k, 2}^{S O}\right| j\right\rangle\right|^{2}}{E_{j}^{(0)}-E_{i}^{(0)}},
$$

with $|i\rangle \equiv\left|n^{\prime} k \eta^{\prime}\right\rangle$, since the perturbation is diagonal in $k$. Now both SO terms contribute. We distinguish the different cases.

(a) $|i\rangle=\left|n k \eta^{\prime}\right\rangle$, with $\eta^{\prime}=-\eta$. In this case, $\left\langle i\left|H_{k, 2}^{S O}\right| j\right\rangle=0$ and we have

$$
E_{j}^{(0)}-E_{i}^{(0)}=\eta\left(\frac{l_{0}}{l_{Z}}\right)^{2}
$$

and 


$$
\begin{aligned}
\left\langle i\left|H_{k, 1}^{S O}\right| j\right\rangle= & \left(\frac{1}{\sqrt{2}}\right)^{2} \frac{k l_{0}^{2}}{2}\left(1-\eta e^{-i \phi_{B}}\right)\left(\begin{array}{cc}
0 & \tilde{l}_{D}+i \tilde{l}_{R} \\
\tilde{l}_{D}-i \tilde{l}_{R} & 0
\end{array}\right) \\
& \times\left(\begin{array}{c}
1 \\
\eta e^{i \phi_{B}}
\end{array}\right) \\
= & \frac{k l_{0}^{2}}{2} \eta i\left(\tilde{l}_{D} \sin \phi_{B}+\tilde{l}_{R} \cos \phi_{B}\right) .
\end{aligned}
$$

This yields

$$
E_{j, a}^{(2)}=\eta \frac{k^{2} l_{0}^{4}}{4}\left(\frac{l_{Z}}{l_{0}}\right)^{2}\left(\tilde{l}_{D} \sin \phi_{B}+\tilde{l}_{R} \cos \phi_{B}\right)^{2} .
$$

(b) $|i\rangle=\left|n^{\prime} k \eta\right\rangle$ with $n^{\prime} \neq n$. Now, $\left\langle i\left|H_{k, 1}^{S O}\right| j\right\rangle=0$ and

$$
E_{j}^{(0)}-E_{i}^{(0)}=n-n^{\prime}
$$

yielding

$$
\begin{aligned}
E_{j, b}^{(2)} & =\sum_{i \neq j} \frac{\left|\left\langle i\left|H_{k, 2}^{S O}\right| j\right\rangle\right|^{2}}{E_{j}^{(0)}-E_{i}^{(0)}} \\
& =-\mid \frac{i l_{0}}{2 \sqrt{2} \cdot 2}\left(\left.1 \quad \eta e^{\left.-i \phi_{B}\right)}\left(\begin{array}{cc}
0 & \tilde{l}_{R}+i \widetilde{l}_{D} \\
\tilde{l}_{R}-i \tilde{l}_{D} & 0
\end{array}\right)\left(\begin{array}{c}
1 \\
\eta e^{i \phi_{B}}
\end{array}\right)\right|^{2}\right. \\
& =-\frac{l_{0}^{2}}{8}\left(\widetilde{l}_{D} \sin \phi_{B}-\widetilde{l}_{R} \cos \phi_{B}\right)^{2} .
\end{aligned}
$$

(c) $|i\rangle=\left|n^{\prime} k \eta^{\prime}\right\rangle$ with $n^{\prime} \neq n$ and $\eta^{\prime}=-\eta$. Again, $\left\langle i\left|H_{k, 1}^{S O}\right| j\right\rangle$ $=0$ and

$$
E_{j}^{(0)}-E_{i}^{(0)}=n-n^{\prime}+\eta\left(\frac{l_{0}}{l_{Z}}\right)^{2}
$$

yielding

$$
\begin{aligned}
& E_{j, c}^{(2)}=\sum_{i \neq j} \frac{\left|\left\langle i\left|H_{k, 2}^{S O}\right| j\right\rangle\right|^{2}}{E_{j}^{(0)}-E_{i}^{(0)}} \\
& =\mid \frac{i l_{0}}{2 \sqrt{2} \cdot 2}\left(1-\eta e^{-i \phi_{B}}\right)\left(\begin{array}{cc}
0 & \tilde{l}_{R}+i \tilde{l}_{D} \\
\tilde{l}_{R}-i \tilde{l}_{D} & 0
\end{array}\right) \\
& \times\left.\left(\begin{array}{c}
1 \\
\eta e^{i \phi_{B}}
\end{array}\right)\right|^{2}\left[\frac{n+1}{-1+\eta\left(l_{0} / l_{Z}\right)^{2}}+\frac{n}{1+\eta\left(l_{0} / l_{Z}\right)^{2}}\right] \\
& =-\frac{l_{0}^{2}}{8}\left(\tilde{l}_{R} \sin \phi_{B}+\tilde{l}_{D} \cos \phi_{B}\right)^{2}\left[\frac{1+\eta\left(l_{0} / l_{Z}\right)^{2}(2 n+1)}{1-\left(l_{0} / l_{Z}\right)^{4}}\right] .
\end{aligned}
$$

The total second-order correction is therefore

$$
\begin{aligned}
E_{n k \eta}^{(2)}= & E_{j, a}^{(2)}+E_{j, b}^{(2)}+E_{j, c}^{(2)} \\
= & \eta \frac{k^{2} l_{0}^{4}}{4}\left(\frac{l_{Z}}{l_{0}}\right)^{2}\left(\widetilde{l}_{D} \sin \phi_{B}+\widetilde{l}_{R} \cos \phi_{B}\right)^{2}-\frac{l_{0}^{2}}{8}\left[\widetilde{l}_{R}^{2}+\widetilde{l}_{D}^{2}\right. \\
& \left.+\left(\tilde{l}_{R} \sin \phi_{B}+\tilde{l}_{D} \cos \phi_{B}\right)^{2} \frac{\left(l_{0} / l_{Z}\right)^{4}+\eta\left(l_{0} / l_{Z}\right)^{2}(2 n+1)}{1-\left(l_{0} / l_{Z}\right)^{4}}\right],
\end{aligned}
$$

showing that both SO terms are entangled and similarly contribute at any angle. Setting $\lambda_{D}=0$, we recover the result of Ref. 4.
${ }^{1}$ A. V. Moroz and C. H. W. Barnes, Phys. Rev. B 60, 14272 (1999)

${ }^{2}$ F. Mireles and G. Kirczenow, Phys. Rev. B 64, 024426 (2001).

${ }^{3}$ M. Governale and U. Zülicke, Phys. Rev. B 66, 073311 (2002).

${ }^{4}$ L1. Serra, D. Sánchez, and R. López, Phys. Rev. B 72, 235309 (2005).

${ }^{5}$ J. Knobbe and Th. Schäpers, Phys. Rev. B 71, 035311 (2005).

${ }^{6}$ S. Debald and B. Kramer, Phys. Rev. B 71, 115322 (2005).

${ }^{7}$ S. Zhang, R. Liang, E. Zhang, L. Zhang, and Y. Liu, Phys. Rev. B 73, 155316 (2006).

${ }^{8}$ F. Malet, M. Pi, M. Barranco, and E. Lipparini, Phys. Rev. B 72, 205326 (2005).

${ }^{9}$ Y. V. Pershin, J. A. Nesteroff, and V. Privman, Phys. Rev. B 69, 121306(R) (2004).

${ }^{10}$ Handbook of Mathematical Functions, edited by M. Abramowitz and I. A. Stegun (Dover, New York, 1972).

${ }^{11}$ V. Gudmundsson, A. Brataas, P. Grambow, B. Meurer, T. Kurth, and D. Heitmann, Phys. Rev. B 51, 17744 (1995).

${ }^{12}$ S. M. Reimann, M. Koskinen, and M. Manninen, Phys. Rev. B 59, 1613 (1999).

${ }^{13}$ O. Heinonen, J. M. Kinaret, and M. D. Johnson, Phys. Rev. B 59, 8073 (1999).
${ }^{14}$ C. A. Ullrich and M. E. Flatté, Phys. Rev. B 66, 205305 (2002).

${ }^{15}$ B. Tanatar and D. M. Ceperley, Phys. Rev. B 39, 5005 (1989).

${ }^{16}$ U. von Barth and L. Hedin, J. Phys. C 5, 1629 (1972).

${ }^{17}$ C. Attaccalite, S. Moroni, P. Gori-Giorgi, and G. B. Bachelet, Phys. Rev. Lett. 88, 256601 (2002); 91, 109902(E) (2003).

${ }^{18}$ G. Vignale and M. Rasolt, Phys. Rev. B 37, 10685 (1988).

${ }^{19}$ M. Ferconi and G. Vignale, Phys. Rev. B 50, 14722 (1994).

${ }^{20}$ M. Pi, M. Barranco, A. Emperador, E. Lipparini, and Ll. Serra, Phys. Rev. B 57, 14783 (1998).

${ }^{21}$ J. C. Lin and G. Y. Guo, Phys. Rev. B 65, 035304 (2001).

${ }^{22}$ S. M. Reimann and M. Manninen, Rev. Mod. Phys. 74, 1283 (2002).

${ }^{23}$ F. Ancilotto, D. G. Austing, M. Barranco, R. Mayol, K. Muraki, M. Pi, S. Sasaki, and S. Tarucha, Phys. Rev. B 67, 205311 (2003).

${ }^{24}$ J. B. Miller, D. M. Zumbuhl, C. M. Marcus, Y. B. Lyanda-Geller, D. Goldhaber-Gordon, K. Campman, and A. C. Gossard, Phys. Rev. Lett. 90, 076807 (2003).

${ }^{25}$ E. Shafir, M. Shen, and S. Saikin, Phys. Rev. B 70, 241302(R) (2004).

${ }^{26}$ J. Könemann, R. J. Haug, D. K. Maude, V. I. Fal'ko, and B. L. Altshuler, Phys. Rev. Lett. 94, 226404 (2005). 
${ }^{27}$ E. Lipparini, Modern Many-Particle Physics (World Scientific, Singapore, 2003).

${ }^{28}$ J. Schliemann, J. C. Egues, and D. Loss, Phys. Rev. Lett. 90, 146801 (2003).

${ }^{29}$ D. Pines and P. Nozières, The Theory of Quantum Liquids (Benjamin, New York, 1966).

${ }^{30}$ K. J. Thomas, D. L. Sawkey, M. Pepper, W. R. Tribe, I. Farrer,
M. Y. Simmons, and D. A. Ritchie, J. Phys.: Condens. Matter 16, L279 (2004).

${ }^{31}$ K. J. Thomas, J. T. Nicholls, M. Y. Simmons, M. Pepper, D. R. Mace, and D. A. Ritchie, Phys. Rev. Lett. 77, 135 (1996).

${ }^{32}$ D. J. Reilly, G. R. Facer, A. S. Dzurak, B. E. Kane, R. G. Clark, P. J. Stiles, J. L. O'Brien, N. E. Lumpkin, L. N. Pfeiffer, and K. W. West, Phys. Rev. B 63, 121311(R) (2001). 